$\underline{\text { Preprint typeset in JHEP style. - HYPER VERSION }}$

Fermilab-Pub-00/134-T

hep-ph/0008192

August 17, 2000

\title{
Light Axion within the Next-to-Minimal Supersymmetric Standard Model
}

\author{
Bogdan A. Dobrescu* and Konstantin T. Matchev ${ }^{\dagger}$ \\ Theoretical Physics Department \\ Fermi National Accelerator Laboratory \\ P.O. Box 500, Batavia, Illinois 60510, USA
}

\begin{abstract}
We analyze the Higgs sector in the Next-to-Minimal Supersymmetric Standard Model, emphasizing the possibility of a light CP-odd scalar (axion) in the spectrum. We compute the coupling of the Standard-Model-like Higgs boson to a pair of axions, and show that it can be large enough to modify the Higgs branching fractions, with a significant impact on the Higgs searches. We delineate the range of parameters relevant for this scenario, and also derive analytic expressions for the scalar masses and couplings in two special cases - a decoupling limit where all scalars other than the axion are heavier than the Standard-Model-like Higgs boson, and the large $\tan \beta$ limit.
\end{abstract}

Keywords: Higgs Physics, Supersymmetric Standard Model.

\footnotetext{
${ }^{*}$ E-mail: bdob@fnal.gov

${ }^{\dagger}$ E-mail: matchev@fnal.gov
} 


\section{Contents}

1. Introduction 1

2. Next-to-Minimal Supersymmetric Standard Model 2

3. The Case of a Light Axion 6

3.1 Approximate $R$-symmetry 6

3.2 Properties of the axion 7

4. The Decoupling Limit, $s \gg v$

5. The Large $\tan \beta$ Limit 13

6. Numerical Results 14

6.1 Collider constraints 14

6.2 Theoretical prejudice 20

7. Conclusions 21

\section{Introduction}

In spite of the phenomenological success of the Standard Model (SM), new physics is expected to appear at some higher energy scale, and hopefully to provide a solution to the hierarchy problem, the origin of fermion masses and CP-violation, and other theoretical puzzles. The new physics may change the properties of the Higgs boson, with a substantial impact for Higgs searches in collider experiments. This is the case in extensions of the SM that include gauge singlet scalars, leading to Higgs boson decays into pairs of light neutral scalars [1]].

Here we point out that the Higgs sector of the Next-to-Minimal Supersymmetric Standard Model (NMSSM) [2] includes an axion, i.e., a pseudo-Nambu-Goldstone boson associated with an anomalous $U(1)$ symmetry, which for a range of parameters is significantly lighter than the other scalars. The NMSSM is a well motivated candidate for physics beyond the Standard Model. Not only does it provide a solution to the hierarchy problem when combined with dynamical supersymmetry breaking, 
but it is also free of the $\mu$ problem that plagues the minimal supersymmetric extension of the SM (MSSM).

In what follows we shall investigate in some detail the Higgs spectrum and couplings in the NMSSM. The purpose of our study is twofold. First, we seek to delineate the region of the NMSSM parameter space consistent with the existing collider data which exhibits a light axion. Second, for the part of parameter space in question, we compute the strength of the SM-like Higgs boson coupling to axion pairs. If this coupling is sizable, it will have a profound effect on the collider searches for the Higgs boson, as the Higgs boson then decays mainly into light axion pairs, and the $b \bar{b}$ signature is diluted.

The Higgs boson decay to axions persists even when the scale of supersymmetry is very large and the superpartners decouple. Furthermore, this phenomenon may occur in non-supersymmetric theories, e.g., composite Higgs models [3, 1] or Majoron models 4 .

The plan of the paper is as follows. In Section 2 we introduce our notation, derive the general tree-level mass matrices of the Higgs sector, and list the trilinear couplings between one CP-even and two CP-odd Higgs bosons in the NMSSM. In Section 3 we concentrate on the case of a light axion, identifying the relevant range of NMSSM parameters and discussing the resulting masses and mixings in the CP-odd scalar sector. We then derive simple analytic expressions for the Higgs spectrum and couplings in two cases of interest - a decoupling limit (Section \) and large $\tan \beta$ (Section 5). A more generic case requires a numerical study, the results being presented in Section 6. Section 7 is reserved for our conclusions.

\section{Next-to-Minimal Supersymmetric Standard Model}

The Next-to-Minimal Supersymmetric Standard Model has the field content of the MSSM with the addition of a gauge-singlet chiral superfield ${ }^{1}, \hat{S}$. In addition to the usual Yukawa-type couplings of the Higgs superfields, $\hat{H}_{u}$ and $\hat{H}_{d}$, to the three generations of quark and lepton superfields, the superpotential $W$ also includes the following terms involving $\hat{S}$ :

$$
W=\lambda \hat{H}_{u} \hat{H}_{d} \hat{S}+\frac{\kappa}{3} \hat{S}^{3}
$$

We assume that the $R$-parity violating terms involving quarks and leptons, and the dimensionful $\hat{H}_{u} \hat{H}_{d}, \hat{S}^{2}$ and $\hat{S}$ terms are forbidden by a gauge symmetry which is spontaneously broken above the TeV scale [5]. A sector of dynamical supersymmetry

\footnotetext{
${ }^{1}$ We shall use hatted symbols to represent chiral superfields and symbols without hats for their lowest (scalar) components.
} 
breaking is supposed to induce masses for the squarks, sleptons and gauginos, as well as soft supersymmetry breaking terms involving the scalar components of $\hat{H}_{u}, \hat{H}_{d}$ and $\hat{S}$ :

$$
V_{\text {soft }}=M_{H_{u}}^{2}\left|H_{u}\right|^{2}+M_{H_{d}}^{2}\left|H_{d}\right|^{2}+M_{S}^{2}|S|^{2}+\sqrt{2}\left(m_{\lambda} H_{u}^{\top} i \sigma_{2} H_{d} S-\frac{m_{\kappa}}{3} S^{3}+\text { h.c. }\right) \text {. }
$$

The $H_{u} H_{d}, S^{2}$ and $S$ soft terms are forbidden by the same symmetry which prevents the $\hat{H}_{u} \hat{H}_{d}, \hat{S}^{2}$ and $\hat{S}$ terms in the superpotential.

In what follows we treat the NMSSM as a low-energy effective field theory valid below some scale, say in the TeV range. Therefore, we define the five mass parameters from $V_{\text {soft }}$ as free parameters at the electroweak scale, $v \approx 246 \mathrm{GeV}$. Phenomenologically, they are constrained by the requirement of having an electroweak asymmetric vacuum and a spectrum of scalars heavier than the current experimental bounds.

The scalar potential for the Higgs sector of the NMSSM is

$$
V=\left|\lambda H_{u}^{\top} i \sigma_{2} H_{d}+\kappa S^{2}\right|^{2}+\lambda^{2}\left(\left|H_{u}\right|^{2}+\left|H_{d}\right|^{2}\right)|S|^{2}+V_{D}+V_{\text {soft }},
$$

with the usual $D$-term contributions

$$
V_{D}=\frac{M_{Z}^{2}}{2 v^{2}}\left(\left|H_{u}\right|^{2}-\left|H_{d}\right|^{2}\right)^{2}+2 \frac{M_{W}^{2}}{v^{2}}\left|H_{u}^{\dagger} H_{d}\right|^{2},
$$

where $M_{W}\left(M_{Z}\right)$ is the $W$-boson $(Z$-boson) mass. If $V$ has a minimum where the vacuum expectation values $(\mathrm{VEVs})$ of $H_{u}$ and $H_{d}$ are aligned and non-zero, the electroweak symmetry is spontaneously broken. Then, in addition to the longitudinal $W$ and $Z$ (i.e. the Nambu-Goldstone modes $G^{+}$and $G^{0}$, respectively) the scalar spectrum includes a charged Higgs boson, $H^{ \pm}$, and five neutral states. In general all five neutral states mix. However, if $\operatorname{Im}\left(\lambda \kappa^{*} m_{\lambda} m_{\kappa}^{*}\right) \ll v^{2}$, then the scalar potential is approximately CP invariant. We will assume that this is the case, so that the mixing of the CP-even neutral scalars with the CP-odd ones can be ignored. It is convenient to derive the spectrum using the basis where the CP-even, $h_{v}^{0}, H_{v}^{0}, h_{s}^{0}$, and the CP-odd, $A_{v}^{0}, A_{s}^{0}$, states are defined as follows:

$$
\begin{aligned}
& H_{d}=\left(\begin{array}{c}
\frac{1}{\sqrt{2}}\left[\left(v+h_{v}^{0}-i G^{0}\right) \cos \beta-\left(H_{v}^{0}-i A_{v}^{0}\right) \sin \beta\right] \\
-G^{-} \cos \beta+H^{-} \sin \beta
\end{array}\right), \\
& H_{u}=\left(\begin{array}{c}
G^{+} \sin \beta+H^{+} \cos \beta \\
\frac{1}{\sqrt{2}}\left[\left(v+h_{v}^{0}+i G^{0}\right) \sin \beta+\left(H_{v}^{0}+i A_{v}^{0}\right) \cos \beta\right]
\end{array}\right) \\
& S=\frac{1}{\sqrt{2}}\left(s+h_{s}^{0}+i A_{s}^{0}\right)
\end{aligned}
$$


with $\tan \beta \equiv\left\langle H_{u}^{2}\right\rangle /\left\langle H_{d}^{1}\right\rangle$. Notice that in order to generate masses for both up-type and down-type quarks one needs VEVs for both $H_{u}$ and $H_{d}$. The $m_{\lambda} H_{u}^{\top} i \sigma_{2} H_{d} S$ soft term forces $S$ to have a non-zero $\mathrm{VEV}, s$. We choose $0<\beta<\pi$, which requires $s>0$ in order to minimize the first term in Eq. (2.3).

There are several advantages of using the basis (2.5) from the beginning. First, notice that $h_{v}^{0}$ is rotated in the same way as $v$, and is exactly the linear combination of $H_{d}^{1}$ and $H_{u}^{2}$ responsible for the masses of the $W$ and $Z$ gauge bosons. Consequently, $h_{v}^{0}$ is also the state which has trilinear couplings to $W$ and $Z$ pairs, and can be produced in association with a $W$ or $Z$ at the Tevatron or LEP. In short, $h_{v}^{0}$ can be identified with the SM-like Higgs boson, $h^{0}$. In addition, the Nambu-Goldstone modes $G^{ \pm}$and $G^{0}$ decouple from the corresponding mass matrices and need not be considered in our further analysis. (The basis (2.5) was considered also in [6]; for results in the more conventional basis see, e.g. [7].)

The extremization conditions for the scalar potential allow us to replace the three mass-squared parameters from $V_{\text {soft }}$ by the three VEVs, $v \sin \beta, v \cos \beta, s$ :

$$
\begin{aligned}
M_{H_{d}}^{2} & =-\frac{\lambda^{2}}{2}\left(s^{2}+v^{2} \sin ^{2} \beta\right)+\frac{\lambda \kappa}{2} s^{2} \tan \beta-\frac{M_{Z}^{2}}{2} \cos 2 \beta+m_{\lambda} s \tan \beta \\
M_{H_{u}}^{2} & =-\frac{\lambda^{2}}{2}\left(s^{2}+v^{2} \cos ^{2} \beta\right)+\frac{\lambda \kappa s^{2}}{2 \tan \beta}+\frac{M_{Z}^{2}}{2} \cos 2 \beta+\frac{m_{\lambda} s}{\tan \beta} \\
M_{S}^{2} & =-\frac{\lambda^{2}}{2} v^{2}+\frac{\lambda \kappa}{2} v^{2} \sin 2 \beta-\kappa^{2} s^{2}+\frac{m_{\lambda} v^{2}}{2 s} \sin 2 \beta+m_{\kappa} s
\end{aligned}
$$

Therefore, the scalar masses depend on the following six unknown parameters: $\tan \beta$, $\lambda, \kappa, s, m_{\lambda}, m_{\kappa}$.

The squared-mass matrix for the CP-even scalars, $h_{v}^{0}, H_{v}^{0}, h_{s}^{0}$, is given by

$$
\mathcal{M}_{h}^{2}=v^{2}\left(\begin{array}{ccc}
r+\frac{M_{Z}^{2}}{v^{2}} & r \cot 2 \beta & \lambda^{2} \frac{s}{v}-R \\
r \cot 2 \beta & -r+\frac{\lambda \kappa s^{2}+2 m_{\lambda} s}{v^{2} \sin 2 \beta} & -R \cot 2 \beta \\
\lambda^{2} \frac{s}{v}-R & -R \cot 2 \beta & \frac{s}{v^{2}}\left(2 \kappa^{2} s-m_{\kappa}\right)+\frac{m_{\lambda}}{2 s} \sin 2 \beta
\end{array}\right)
$$

where we have defined

$$
r \equiv\left(\frac{\lambda^{2}}{2}-\frac{M_{Z}^{2}}{v^{2}}\right) \sin ^{2} 2 \beta
$$

and

$$
R \equiv \frac{1}{v}\left(\lambda \kappa s+m_{\lambda}\right) \sin 2 \beta
$$


We label the CP-even mass eigenstates in order of increasing mass,

$$
\left(\begin{array}{c}
H_{1}^{0} \\
H_{2}^{0} \\
H_{3}^{0}
\end{array}\right)=U\left(\begin{array}{c}
h_{v}^{0} \\
H_{v}^{0} \\
h_{s}^{0}
\end{array}\right)
$$

where the $3 \times 3$ orthogonal matrix $U$ may be obtained by diagonalizing $\mathcal{M}_{h}^{2}$. Whenever there is no confusion, we shall use an alternative labelling of the Higgs boson mass eigenstates, in analogy to the MSSM. We shall use $h^{0}$ for the SM-like mass state, i.e. the one with the largest projection onto $h_{v}^{0} ; H^{0}$ for the state corresponding to the "heavy" CP-even Higgs boson of the MSSM, i.e. the one with the largest projection onto $H_{v}^{0}$; and $H^{\prime 0}$ for the state corresponding to the additional singlet of the NMSSM, i.e. the one with the largest projection onto $h_{s}^{0}$. As can be readily seen from (2.7), in the limit of large $s$, the SM-like Higgs boson $h^{0}$ is identified with $H_{1}^{0}$. However, for small values of $s, h^{0}$ can also be $H_{2}^{0}$ or even $H_{3}^{0}$, depending on the other parameters.

The CP-odd states, $A_{v}^{0}, A_{s}^{0}$ have the following squared-mass matrix:

$$
\mathcal{M}_{A}^{2}=\left(\begin{array}{cc}
\frac{\lambda \kappa s^{2}+2 m_{\lambda} s}{\sin 2 \beta} & -v\left(\lambda \kappa s-m_{\lambda}\right) \\
-v\left(\lambda \kappa s-m_{\lambda}\right) & \left(\lambda \kappa+\frac{m_{\lambda}}{2 s}\right) v^{2} \sin 2 \beta+3 s m_{\kappa}
\end{array}\right) .
$$

The CP-odd mass eigenstates may be written in terms of the $A_{v}^{0}$ and $A_{s}^{0}$ states:

$$
\left(\begin{array}{c}
A_{1}^{0} \\
A_{2}^{0}
\end{array}\right)=\left(\begin{array}{cc}
\cos \theta_{A} & \sin \theta_{A} \\
-\sin \theta_{A} & \cos \theta_{A}
\end{array}\right)\left(\begin{array}{c}
A_{v}^{0} \\
A_{s}^{0}
\end{array}\right)
$$

with a mixing angle $\theta_{A}$ that satisfies

$$
\tan 2 \theta_{A}=\frac{-4 v s\left(\lambda \kappa s-m_{\lambda}\right) \sin 2 \beta}{v^{2} \sin ^{2} 2 \beta\left(2 \lambda \kappa s+m_{\lambda}\right)-2 s^{2}\left(\lambda \kappa s+2 m_{\lambda}-3 m_{\kappa} \sin 2 \beta\right)} .
$$

Finally, the charged Higgs boson has a mass

$$
M_{H^{ \pm}}^{2}=\frac{\lambda \kappa s^{2}+2 m_{\lambda} s}{\sin 2 \beta}-\frac{\lambda^{2}}{2} v^{2}+M_{W}^{2} .
$$

The vacuum defined by Eq. (2.5) is indeed a viable minimum of the scalar potential provided all physical scalars have positive masses. Therefore, all eigenvalues of $\mathcal{M}_{h}^{2}$ and $\mathcal{M}_{A}^{2}$ have to be positive, and $M_{H^{ \pm}}^{2}>0$. We will analyze the constraints on the parameter space imposed by these conditions both numerically (Section 6) and analytically in certain interesting limits (Sections 4 and 5).

In particular, we will be concentrating on the case where one of the CP-odd scalars is light, and therefore the neutral CP-even scalars may decay into a pair 
of CP-odd states. The relevant trilinear couplings in the basis (2.5) are given at tree-level by

$$
\begin{aligned}
\mathcal{L}_{H A A}=-\frac{v}{2}\{ & {\left[\left(\frac{\lambda^{2}}{2}+r \cot ^{2} 2 \beta\right) h_{v}^{0}-r \cot 2 \beta H_{v}^{0}+\left(R+\lambda^{2} \frac{s}{v}\right) h_{s}^{0}\right]\left(A_{v}^{0}\right)^{2} } \\
& +\left[\lambda(\kappa \sin 2 \beta+\lambda) h_{v}^{0}+\lambda \kappa \cos 2 \beta H_{v}^{0}+2 \frac{\kappa^{2} s-m_{\kappa}}{v} h_{s}^{0}\right]\left(A_{s}^{0}\right)^{2} \\
& \left.-2\left(\frac{\lambda \kappa s-m_{\lambda}}{v} h_{v}^{0}+\lambda \kappa h_{s}^{0}\right) A_{v} A_{s}\right\} .
\end{aligned}
$$

In order to compute the Higgs decay width into CP-odd scalars, one has first to determine the rotation matrix $U$ and the mixing angle $\theta_{A}$, and then to derive the trilinear couplings in the mass eigenstate basis. We will perform this computation in the following sections.

\section{The Case of a Light Axion}

\subsection{Approximate $R$-symmetry}

The scalar potential $V$ has no global continuous symmetry. However, in the limit where the coefficients of the trilinear terms vanish, $m_{\lambda}, m_{\kappa} \rightarrow 0$, the potential has a global $U(1)_{R}$ symmetry under which the $S$ charge, $y_{S} \neq 0$, is half the charge of $H_{u} H_{d}$. This symmetry is spontaneously broken by the VEVs of $H_{u}, H_{d}$ and $S$, so that apparently there is a Nambu-Goldstone boson in the spectrum. In addition, $U(1)_{R}$ is explicitly broken by the QCD anomaly. To see this, note that the Yukawa terms responsible for quark masses impose constraints on the $U(1)_{R}$ charges of the quarks such that the $\left[S U(3)_{C}\right]^{2} \times U(1)_{R}$ anomaly is proportional to $y_{S}$. Hence the Nambu-Goldstone boson is in fact an axion, and there is a small contribution to its mass from QCD.

Furthermore, there is another source of explicit $U(1)_{R}$ breaking. To see this, note that the form of the superpotential (2.1) requires that $U(1)_{R}$ does not commute with supersymmetry, i.e. the fermion components of the $\hat{H}_{u}, \hat{H}_{d}$ and $\hat{S}$ superfields have different $U(1)_{R}$ charges than the corresponding scalars. Therefore, this $U(1)_{R}$ is an $R$ symmetry. Given that the gauginos carry $R$-charge, it follows that $U(1)_{R}$ is explicitly broken by the gaugino masses. This effect appears in the effective potential via oneloop diagrams with $H_{u, d}$-gaugino-Higgsino vertices. Although this contribution to the axion mass is larger than the contribution from the anomaly, the loop suppression implies that the axion is lighter than the other scalars by more than an order of magnitude in the limit $m_{\lambda}, m_{\kappa} \rightarrow 0$. 
One has to be alert for potential confusions regarding the "axion" label used in this paper. This axion is not useful for solving the strong CP problem because the explicit $U(1)_{R}$ breaking due to gaugino masses exceeds the anomaly contribution. Also, the axion associated with this approximate $U(1)_{R}$ is different from the PecceiQuinn axion associated with the global $U(1)$ recovered in the $\kappa, m_{\kappa} \rightarrow 0$ limit.

Another confusion may be caused by the $R$-axion from the dynamical supersymmetry breaking sector. Typically, the models of dynamical supersymmetry breaking have a spontaneously broken $R$-symmetry [8]. The associated Nambu-Goldstone boson is called an $R$-axion and would be massless in the absence of a source of $R$ symmetry breaking, such as a term in the superpotential required for cancelling the cosmological constant [9]. If there is a hidden sector where supersymmetry is dynamically broken, and supersymmetry breaking is mediated from this sector to the NMSSM via supergravity, gauge interactions, or any other mechanism, then there is mixing between the $R$-axion and the axion discussed in this paper. However, this mixing is suppressed by the scale associated with supersymmetry breaking mediation, and may be ignored for practical purposes. It is important however, that the spontaneous breaking of the $R$-symmetry within the hidden sector is the source of gaugino masses in the NMSSM. Therefore, the existence of the $R$-axion in the hidden sector requires a mass for the $U(1)_{R}$ axion from the NMSSM.

\subsection{Properties of the axion}

The light axion may also be identified by studying the spectrum of CP-odd states given in Section 2. In what follows we will expand in $m_{\lambda} / v$ and $m_{\kappa} / v$, neglecting the loop effects, which is appropriate for $1 \gg m_{\lambda, \kappa} / v \gtrsim \mathcal{O}\left(10^{-3}\right)$. The lightest CP-odd neutral scalar, $A_{1}^{0}$, is the axion associated with the approximate $U(1)_{R}$ symmetry, and its mass,

$$
M_{A_{1}}=\sqrt{3 s}\left(m_{\kappa} \sin ^{2} \theta_{A}+\frac{3 m_{\lambda} \cos ^{2} \theta_{A}}{2 \sin 2 \beta}\right)^{1 / 2}+\mathcal{O}\left(m_{\lambda, \kappa}^{3 / 2} / \sqrt{v}\right),
$$

vanishes in the limit $m_{\lambda}, m_{\kappa} \rightarrow 0$, in agreement with the arguments presented above. The other CP-odd neutral scalar, $A_{2}^{0}$, has a mass

$$
M_{A_{2}} \approx \frac{v}{\cos \theta_{A}} \sqrt{\lambda \kappa \sin 2 \beta}+\mathcal{O}\left(m_{\lambda}, m_{\kappa}\right)
$$

The mixing angle $0<\theta_{A}<\pi / 2$ also has a simple form:

$$
\tan \theta_{A}=\frac{s}{v \sin 2 \beta}+\mathcal{O}\left(m_{\lambda, \kappa} / v\right)
$$

Notice that in the small $m_{\lambda}, m_{\kappa}$ limit the axion mass $M_{A_{1}}$ depends on only four out of the six input parameters of the Higgs sector: $s, \tan \beta, m_{\lambda}$ and $m_{\kappa}$. The dependence 
on $\lambda$ and $\kappa$ drops out because they are not related to the breaking of the $U(1)_{R}$ symmetry.

The couplings of the axion to quarks and leptons may easily be derived by observing that its $A_{v}^{0}$ component has the couplings of the MSSM CP-odd scalar while $A_{s}^{0}$ does not couple to the quarks and leptons. Therefore, the couplings are proportional to the fermion masses within the up- and down-type sectors separately:

$$
\frac{\cos \theta_{A}}{v}\left(m_{u} \cot \beta \bar{u} \gamma_{5} u+m_{d} \tan \beta \bar{d} \gamma_{5} d\right) i A_{1}^{0} \text {. }
$$

However, in contrast to the MSSM CP-odd scalar, the couplings of $A_{1}^{0}$ to down-type fermions are not enhanced by $\tan \beta$ because in the large $\tan \beta$ limit Eq. (3.3) gives

$$
\cos \theta_{A} \approx \frac{v}{s} \frac{2}{\tan \beta}
$$

We then see that the $\tan \beta$ enhancement of the $A_{v}^{0}$ coupling to down-type fermions is exactly compensated by a $\tan \beta$ suppression in the mixing angle $\theta_{A}$. On the other hand, the $A_{1}^{0}$ couplings to up-type fermions are doubly suppressed by $\tan \beta$, and Eq. (3.4) becomes

$$
\frac{2}{s}\left(\frac{m_{u}}{\tan ^{2} \beta} \bar{u} \gamma_{5} u+m_{d} \bar{d} \gamma_{5} d\right) i A_{1}^{0} .
$$

The phenomenological implications of this result are clear: when $\tan \beta \gg 1$ the crosssections for $A_{1}^{0}$ production in association with a pair of down-type fermions $(e . g . b \bar{b})$ do not depend on $\tan \beta$, while the $A_{1}^{0}$ branching ratios into down-type fermions are enhanced.

Of special interest for Higgs phenomenology is the axion coupling to the SM-like Higgs boson $h^{0}$,

$$
\mathcal{L}_{h^{0} A_{1}^{0} A_{1}^{0}}=\frac{c}{2} v h^{0} A_{1}^{0} A_{1}^{0},
$$

where

$$
\begin{aligned}
c=\frac{-1}{1+\frac{v^{2}}{s^{2}} \sin ^{2} 2 \beta} & \left\{U_{i 1}\left[\lambda(\lambda-\kappa \sin 2 \beta)+\frac{v^{2}}{s^{2}}\left(\frac{\lambda^{2}}{2} \sin ^{2} 2 \beta+r \cos ^{2} 2 \beta\right)\right]\right. \\
& +U_{i 2} \cos 2 \beta\left(\lambda \kappa-\frac{v^{2}}{s^{2}} r \sin 2 \beta\right) \\
& \left.+U_{i 3} \frac{s}{v}\left[2 \kappa^{2}+\frac{v^{2}}{s^{2}} \lambda \sin 2 \beta\left(\kappa \sin ^{2} 2 \beta-2 \kappa+\lambda \sin 2 \beta\right)\right]\right\} .
\end{aligned}
$$

Here the index $i$ labels the mass eigenstate corresponding to $h^{0}$. Although analytical expressions for the elements of the rotation matrix $U$ may be written in general [10], here we prefer to derive the trilinear coupling $c$ as a series in $v / s$ (Section 4) or $1 / \tan \beta$ (Section 5), which should help the reader gain some insight into the numerical results of Section 6 . 


\section{The Decoupling Limit, $s \gg v$}

In this Section we study the decoupling limit of the NMSSM in which $A_{1}^{0}$ and the lightest $\mathrm{CP}$-even scalar, $H_{1}^{0}$, are much lighter than the other scalars. This situation arises when the gauge-singlet $\mathrm{VEV}$ is large ${ }^{2}, s \gg v$, as can be seen by inspecting the expressions for $\mathcal{M}_{h}^{2}, \mathcal{M}_{A}^{2}$ and $M_{H^{ \pm}}^{2}$ given in Section 2. Under those circumstances, $H_{1}^{0}$ is simply the SM-like Higgs boson $h^{0}$, and the low-energy limit of the model is just the SM, with the addition of one (mostly singlet) CP-odd scalar, $A_{1}^{0}$.

In order to analyze the CP-even neutral states we diagonalize $\mathcal{M}_{h}^{2}$ by expanding in $v / s$. We keep only the leading order in $m_{\lambda, \kappa} / v$ because we will only be interested in the region of parameter space where there is a light axion. The CP-even mass eigenstates are given by Eq. (2.10), with

$$
\begin{aligned}
U_{11}= & 1-\frac{v^{2}}{s^{2}} \frac{\lambda^{2}}{8 \kappa^{4}}(\lambda-\kappa \sin 2 \beta)^{2}+\mathcal{O}\left(\frac{v^{4}}{s^{4}}\right), \\
U_{12}= & -\frac{v^{2}}{s^{2}} \frac{\lambda^{2}}{4 \kappa^{2}}\left(1-\frac{2 \kappa}{\lambda^{3}} \frac{M_{Z}^{2}}{v^{2}} \sin 2 \beta\right) \sin 4 \beta+\mathcal{O}\left(\frac{v^{3}}{s^{3}}\right), \\
U_{13}= & -\frac{v}{s} \frac{\lambda^{2}}{2 \kappa^{2}}\left(1-\frac{\kappa}{\lambda} \sin 2 \beta\right)-\frac{v^{3}}{s^{3}}\left\{\frac{M_{Z}^{2}}{v^{2}} \frac{\cos ^{2} 2 \beta}{4 \kappa^{4}}(\lambda+\kappa \sin 2 \beta)(\lambda-2 \kappa \sin 2 \beta)\right. \\
& \left.-\frac{\lambda^{3}}{4 \kappa^{3}}\left[\frac{\lambda-\kappa \sin 2 \beta}{4 \kappa^{3}}\left(3 \lambda^{2}-6 \lambda \kappa \sin 2 \beta+\kappa^{2} \sin ^{2} 2 \beta\right)-\sin 2 \beta \cos ^{2} 2 \beta\right]\right\} \\
& +\mathcal{O}\left(\frac{v^{4}}{s^{4}}\right) .
\end{aligned}
$$

The other elements of the orthogonal matrix $U$,

$$
\begin{aligned}
U_{21} & =-\frac{v^{2}}{s^{2}} \sin ^{2} 2 \beta \cos 2 \beta\left[\frac{\lambda^{2}}{2 \kappa(\lambda-2 \kappa \sin 2 \beta)}+\frac{M_{Z}^{2}}{\lambda \kappa v^{2}}\right]+\mathcal{O}\left(\frac{v^{3}}{s^{3}}\right), \\
U_{31} & =\frac{v}{s} \frac{\lambda^{2}}{2 \kappa^{2}}\left(1-\frac{\kappa}{\lambda} \sin 2 \beta\right)+\mathcal{O}\left(\frac{v^{3}}{s^{3}}\right) \\
U_{23} & =-U_{32}=-\frac{v}{s} \frac{\lambda \sin 2 \beta \cos 2 \beta}{\lambda-2 \kappa \sin 2 \beta}+\mathcal{O}\left(\frac{v^{3}}{s^{3}}\right) \\
U_{22} & =1-\frac{v^{2}}{2 s^{2}}\left(\frac{\lambda \sin 2 \beta \cos 2 \beta}{\lambda-2 \kappa \sin 2 \beta}\right)^{2}+\mathcal{O}\left(\frac{v^{4}}{s^{4}}\right)
\end{aligned}
$$

\footnotetext{
${ }^{2}$ Note that the dimensionless couplings $\lambda$ and $\kappa$ from the superpotential of the Higgs sector are usually assumed to be roughly of order one, i.e. we ignore cases where a hierarchy is generated through some kind of fine-tuning of the couplings.
} 


$$
U_{33}=1-\frac{v^{2}}{2 s^{2}}\left\{\left(\frac{\lambda \sin 2 \beta \cos 2 \beta}{\lambda-2 \kappa \sin 2 \beta}\right)^{2}+\left[\frac{\lambda(\lambda-\kappa \sin 2 \beta)}{2 \kappa^{2}}\right]^{2}\right\}+\mathcal{O}\left(\frac{v^{4}}{s^{4}}\right)
$$

are less important in what follows, and we only list them here for completeness. Notice the $s / v$ enhancement in the last line of Eq. (3.8), which requires us to compute $U_{13}$ up to an additional order in the $v / s$ expansion.

After computing the eigenvalues of $\mathcal{M}_{h}^{2}$ as a power series in $v / s$, we find the following CP-even scalar masses:

$$
\begin{aligned}
& M_{h^{0}}=v\left[\frac{M_{Z}^{2}}{v^{2}} \cos ^{2} 2 \beta-\frac{\lambda^{3}}{2 \kappa^{2}}(\lambda-2 \kappa \sin 2 \beta)\right]^{1 / 2}+\mathcal{O}\left(\frac{v^{3}}{s^{2}}\right), \\
& M_{H^{0}}=s\left(\frac{\lambda \kappa}{\sin 2 \beta}\right)^{1 / 2}+\mathcal{O}\left(\frac{v^{2}}{s}\right), \\
& M_{H^{\prime 0}}=\sqrt{2} \kappa s+\mathcal{O}\left(\frac{v^{2}}{s}\right) .
\end{aligned}
$$

We see from Eqs. (4.4), (2.11) and (2.14) that to leading order in $v / s$, the $H^{0}, A_{2}^{0}$ and $H^{ \pm}$scalars are degenerate, forming a weak-doublet complex scalar of mass

$$
s\left(\frac{\lambda \kappa}{\sin 2 \beta}\right)^{1 / 2} \gg v \text {. }
$$

These are the familiar "heavy" Higgs bosons of the MSSM. Eq. (4.5) confirms that the $H^{\prime 0}$ scalar is also heavy, with a mass of the order of the gauge singlet VEV $s$. Therefore, in the limit $s \gg v$ considered in this section, the only surviving scalars with masses of order the electroweak scale or smaller are the SM-like Higgs boson $h^{0}$ and the axion $A_{1}^{0}$.

The above discussion is illustrated in Fig. 1, where we plot the exact tree-level masses of the CP-even Higgs bosons (solid lines), the CP-odd Higgs bosons (dashed lines) and the charged Higgs boson (dotted), as a function of $s$, for fixed $\tan \beta=2$, $\lambda=\kappa=0.5$ and $m_{\lambda}=m_{\kappa}=1 \mathrm{GeV}$. In order to guide the eye, we have added shading to trace the SM-like Higgs boson $h^{0}$, which from right to left is identified successively with $H_{1}^{0}, H_{2}^{0}$ and $H_{3}^{0}$.

In the limit $s \gg v$, the condition $M_{h^{0}}^{2}>0$ is necessary and sufficient for the existence of the electroweak asymmetric vacuum described by Eq. (2.5). The resulting constraint on the parameter space is given at tree-level by

$$
\frac{\lambda^{4}}{2 \kappa^{2}}<\frac{M_{Z}^{2}}{v^{2}} \cos ^{2} 2 \beta+\frac{\lambda^{3}}{\kappa} \sin 2 \beta
$$

Alternatively, for $s<v$, we see from Fig. 1 that $H_{1}^{0}=H^{0}$ instead, and the requirement for positivity of the Higgs masses squared implies a lower bound of 


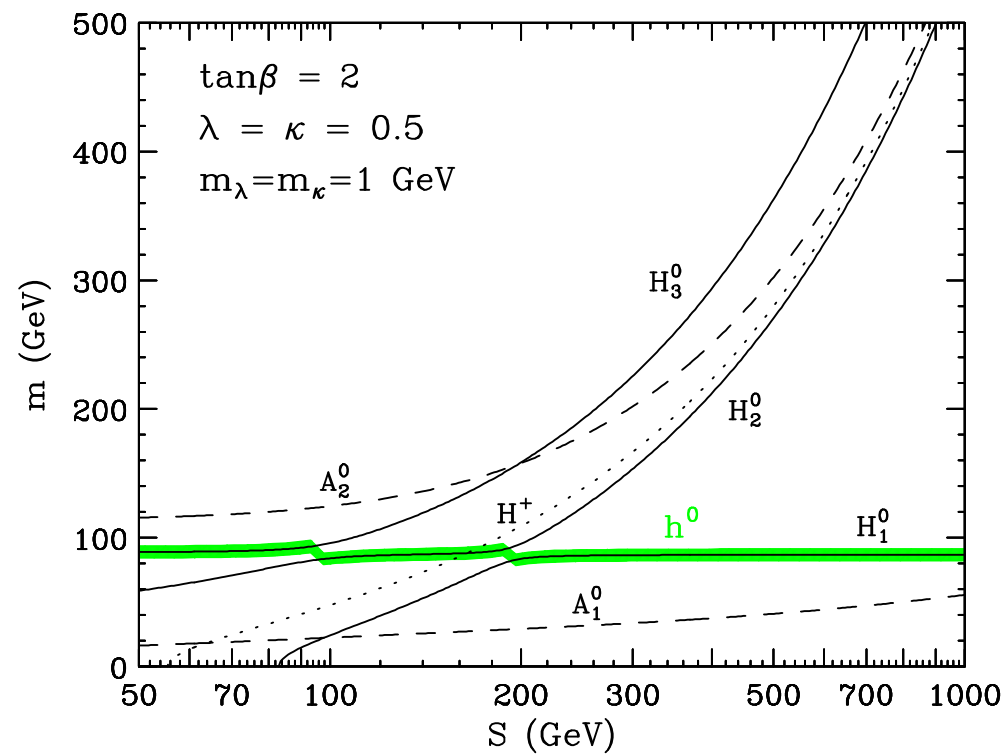

Figure 1: Higgs boson spectrum as a function of $s$, for $\tan \beta=2, \lambda=\kappa=0.5$ and $m_{\lambda}=m_{\kappa}=1 \mathrm{GeV}$.

$s$. In any way, LEP-II bounds on $M_{h^{0}}$ should provide a stronger constraint on the parameters than the requirement of a local minimum of the potential.

Just as an aside, notice that Eq. (4.3) obeys the usual NMSSM tree-level upper bound on the lightest CP-even scalar [11, 12],

$$
M_{H_{1}^{0}}^{2}<M_{Z}^{2} \cos ^{2} 2 \beta+\frac{1}{2} \lambda^{2} v^{2} \sin ^{2} 2 \beta
$$

since Eq. (4.3) can be equivalently rewritten as

$$
M_{h^{0}}^{2}=M_{Z}^{2} \cos ^{2} 2 \beta+\frac{1}{2} \lambda^{2} v^{2} \sin ^{2} 2 \beta-\frac{1}{2} \lambda^{2} v^{2}\left(\sin 2 \beta-\frac{\lambda}{\kappa}\right)^{2} .
$$

In this decoupling limit, $h^{0}$ couples to the quarks and leptons exactly like the Standard Model Higgs boson. Nevertheless, the decays of $h^{0}$ may be very different than in the SM, if $M_{A_{1}}<M_{h} / 2$, since the $h^{0} \rightarrow A_{1}^{0} A_{1}^{0}$ decay mode is then kinematically open. In order to assess the partial width for this decay mode [1],

$$
\Gamma\left(h^{0} \rightarrow A_{1}^{0} A_{1}^{0}\right)=\frac{c^{2} v^{2}}{32 \pi M_{h^{0}}}\left(1-4 \frac{M_{A_{1}}^{2}}{M_{h^{0}}^{2}}\right)^{1 / 2},
$$

we need to compute the coefficient $c$ of the trilinear term $\mathcal{L}_{h^{0}} A_{1}^{0} A_{1}^{0}$ shown in Eq. (3.7). Plugging the elements of $U$ shown in Eqs. (4.1) into Eq. (3.8), after a somewhat tedious calculation we find a simple result:

$$
c=\frac{1}{2 \kappa^{2}}(\lambda-2 \kappa \sin 2 \beta)(\lambda+\kappa \sin 2 \beta) \frac{M_{h}^{2}}{s^{2}}+\mathcal{O}\left(\frac{v^{3}}{s^{3}}\right) .
$$




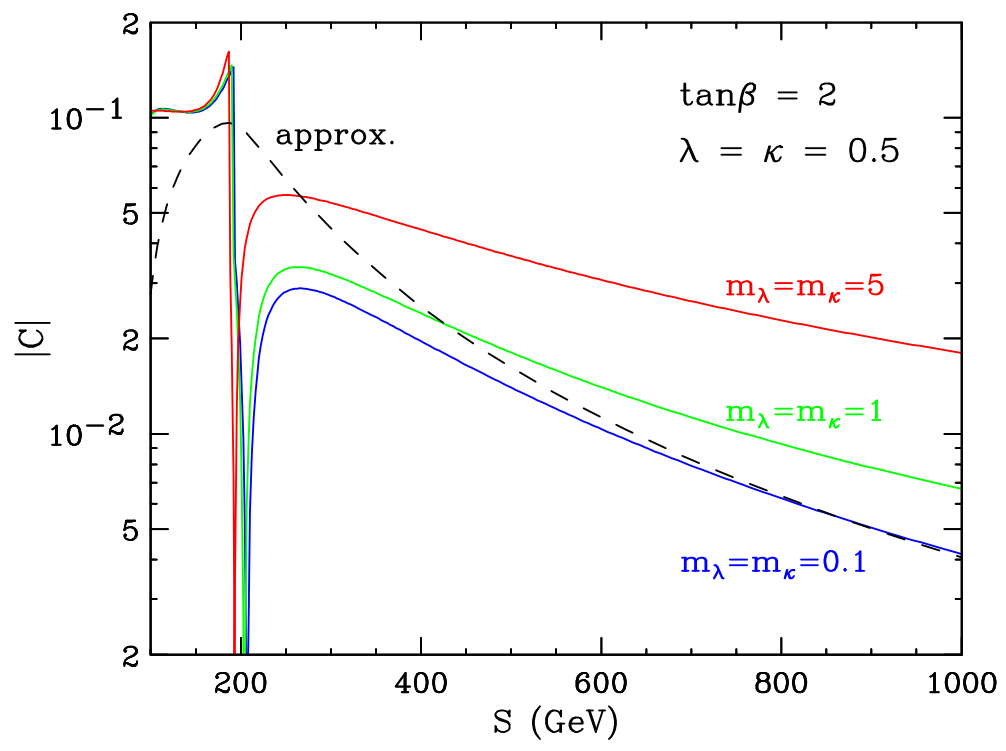

Figure 2: Comparison between the analytic approximation (4.11) (dashed) and the exact results for $|c|$ (solid lines), as a function of $s$, for $\tan \beta=2, \lambda=\kappa=0.5$, and three different values of $m_{\lambda}=m_{\kappa}$ (shown, in $\mathrm{GeV}$ ).

This compact formula has a simple physical interpretation. In the polar coordinates parametrization of the axion field, the axion has only derivative couplings, suppressed by the axion decay constant, $f_{A}$. Since the axion decay constant is given by the $U(1)_{R}$ breaking $\mathrm{VEV}$, in the $s \gg v$ limit we find $f_{A} \approx s$, while the derivatives in the coupling yield a factor of $M_{h^{0}}^{2}$, after taking into account the equations of motion. In addition, the coupling of the Higgs boson to pairs of axions has to be proportional to the Higgs VEV. Therefore, the amplitude for $h^{0} \rightarrow A_{1}^{0} A_{1}^{0}$ is proportional to $v M_{h^{0}}^{2} / s^{2}$. The amplitude has to be the same in the polar and orthogonal coordinates, which explains both the appearance of the Higgs boson mass $M_{h^{0}}$ rather than $v$ in the numerator of Eq. (4.11), as well as the cancellation of the terms of order $(v / s)^{0}$ and $(v / s)^{1}$. In the Appendix we provide the derivation of the Higgs coupling to axion pairs in the polar coordinates parametrization.

Since $\lambda$ and $\kappa$ are expected to be of order one, the size of $c$ is basically dictated by the degree of decoupling of the heavy scalars. The quality of the approximation is shown in Fig. 2, where we compare the prediction of the analytical formula (1.11) (dashed line) with the exact numerical results for the absolute value of the tree-level coefficient $|c|$ (solid lines), as a function of $s$, for $\tan \beta=2, \lambda=\kappa=0.5$, and three different values of $m_{\lambda}=m_{\kappa}$ (shown in $\mathrm{GeV}$ ). We see that as expected, at large $s$ the analytic approximation agrees pretty well with the exact result for the smallest values of $m_{\lambda}$ and $m_{\kappa}$. The approximation fails either at small $s$, or for larger $m_{\lambda}$ and $m_{\kappa}$ - recall that we neglected terms of order $m_{\lambda} / v$ and $m_{\kappa} / v$, while the leading 
term in Eq. (4.11) is of order $v^{2} / s^{2}$. The dip around $s \sim 200 \mathrm{GeV}$ is due to the fact that $c$ changes sign, as we switch from $h^{0}=H_{1}^{0}$ to $h^{0}=H_{2}^{0}$.

\section{The Large $\tan \beta$ Limit}

In this section we shall obtain the coefficient $c$ in the limit of large $\tan \beta$. To this end we follow the procedure from the previous Section - expand $U$ in powers of $1 / \tan \beta$ and substitute the result in Eq. (3.8). However, we will not take the limit $m_{\lambda}, m_{\kappa} \rightarrow 0$, but instead will keep the full dependence on $m_{\lambda}, m_{\kappa}$, as this does not cause too much complication. Keeping only leading order terms, Eq. (3.8) simplifies to

$$
c=-U_{i 1} \lambda^{2}+U_{i 2} \lambda \kappa-U_{i 3} 2 \kappa^{2} \frac{s}{v} .
$$

The relevant mixing angles in the CP-even Higgs sector are also easy to compute -

notice that in the large $\tan \beta$ limit, $H^{0}$ decouples and to leading order $H_{1}^{0}$ is a linear combination of $h_{v}^{0}$ and $h_{s}^{0}$ only. We find

$$
U_{11}=\cos \theta_{H}, \quad U_{12}=0, \quad U_{13}=-\sin \theta_{H},
$$

where

$$
\tan 2 \theta_{H}=\frac{2 \lambda^{2} s v}{2 \kappa^{2} s^{2}-M_{Z}^{2}-m_{\kappa} s} .
$$

The coefficient $c$ is then simply

$$
c=-\lambda^{2} \cos \theta_{H}+2 \kappa^{2} \frac{s}{v} \sin \theta_{H}+\mathcal{O}\left(\frac{1}{\tan \beta}\right) .
$$

In Fig. 3 we show the validity of the approximation (5.4). In analogy to Fig. 2, we plot $|c|$, but this time versus $\tan \beta$, for a fixed value of $s=300 \mathrm{GeV}$. We see reasonable agreement between the exact result and our approximation, given the large cancellations between the leading order terms. We have checked that each individual term contributing to $c$ is reproduced with an accuracy of a few percent, and the remaining difference in the total seen in the Fig. 3 is due to a large cancellation between the first and third terms of Eq. (5.1). Although the analytical approximations presented here are not intended to substitute the full result, they illustrate these leading order cancellations. It is also easy now to understand why $c$ vanishes for a particular value of $\tan \beta$ in Fig. B: for $\sin 2 \beta=\lambda /(2 \kappa)$ the analytical approximation (4.11) gives $c=0$.

In conclusion of this section, we note that when in addition we take the limit of large $s$, Eq. (5.4) reduces to

$$
c=\frac{\lambda^{2}}{2 \kappa^{2}} \frac{M_{h}^{2}}{s^{2}}+\mathcal{O}\left(\frac{v^{3}}{s^{3}}, \frac{1}{\tan \beta}\right),
$$

which is also in agreement with the large $\tan \beta$ limit of Eq. (4.11). 


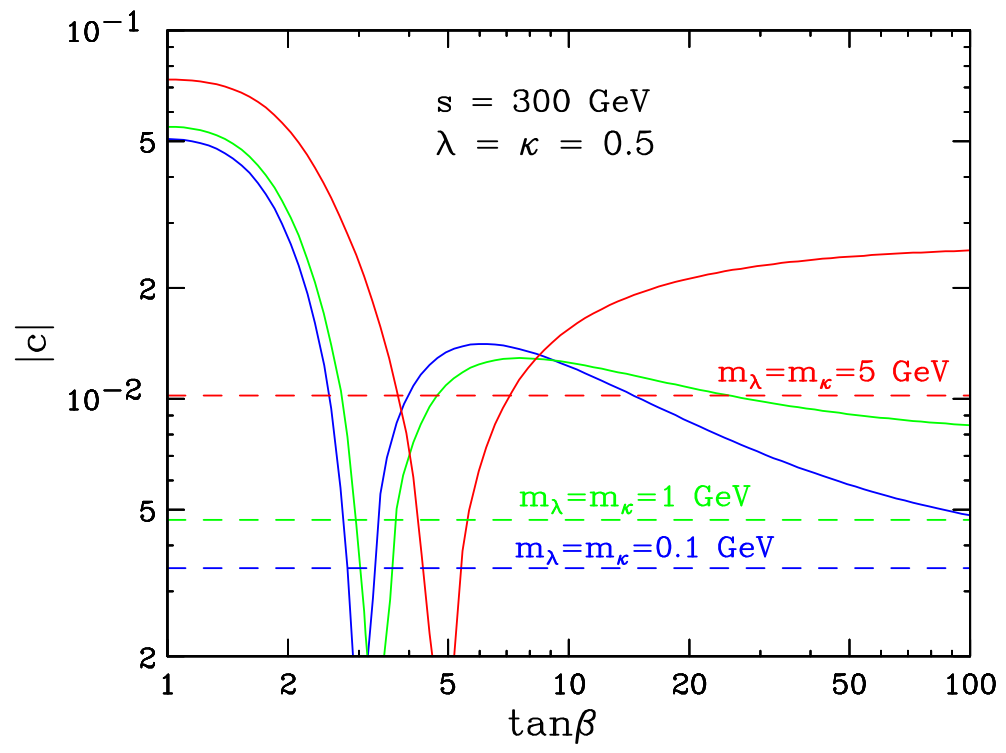

Figure 3: The same as Fig. 2, except plotted as a function of $\tan \beta$, for fixed $s=300$ GeV. Unlike Fig. 2, here there is a different approximation for each value of $m_{\kappa}$, as we kept the full $m_{\kappa}$ dependence in this section.

\section{Numerical Results}

In this section we shall study numerically the scalar spectrum and the coupling (3.7) of the Standard Model-like Higgs boson $h^{0}$ to axion pairs. There are several experimental (Section 6.1) and theoretical (Section 6.2) constraints on our scenario. Most importantly, we must account for all existing experimental bounds on the Higgs bosons and their superpartners. (We do not consider experimental bounds from other superpartner searches, since those depend on the particular framework of supersymmetry breaking, which we never had to specify for our Higgs sector analysis.)

The Higgs structure considered here must eventually be embedded in some more fundamental theory, defined at a much higher scale $\Lambda$, possibly the Planck or the string scale. Hence, the low-energy Higgs sector parameters should be derived in terms of the parameters from the fundamental theory without excessive fine-tuning. It is also theoretically desirable that the couplings in the theory are free of Landau poles at least up to the scale $\Lambda$.

Our numerical analysis in this Section is designed to address these issues.

\subsection{Collider constraints}

Let us first start with the Higgs sector. LEP is typically able to rule out new particles with order one couplings close to its kinematic limit. For example, the charged Higgs 
bosons $H^{ \pm}$can be produced in $s$-channel $Z / \gamma$ processes. The current LEP bound is $m_{H^{+}} \gtrsim 85 \mathrm{GeV}$ [13] and we expect it to be valid in the case of the NMSSM discussed here as well.

The Standard Model Higgs search at LEP, when reinterpreted as a search for the lightest CP-even Higgs boson of the MSSM, provides an additional constraint on our parameter space. The current combined LEP limit is around $113 \mathrm{GeV}$. As is well known however, the one-loop corrections to $M_{h}$ involving third generation quarks and their superpartners are positive and potentially large [14], so that the LEP constraint on the tree-level Higgs boson mass $M_{h}$ is much weaker. Depending on the stop/sbottom masses and the amount of squark mixing, the radiative corrections can shift the tree-level value of the Higgs mass by up to $\Delta M_{h} \sim 20-30 \mathrm{GeV}$ [15]. Hence we shall allow for tree-level Higgs boson masses as low as 80-90 GeV. Of course, within any given supersymmetric model framework, one can compute this difference exactly in terms of the parameters of the squark sector. Here we prefer to stay within our model-independent approach and avoid specifying a particular model of supersymmetry breaking and/or squark spectrum and mixing angles, as this will hinder the universal applicability of our results. Furthermore, the novel effect of $h \rightarrow A_{1}^{0} A_{1}^{0}$ decays is maximally operational for large $h^{0}$ masses (see, e.g. Eq.(5.5)), where the LEP bound is likely to be satisfied.

Finally, LEP has also searched for superpartners of the $S U(2)$ Higgs bosons, as part of their chargino search. Independent of their mass splitting, charged higgsinos lighter than $\sim 72 \mathrm{GeV}$ [16] have been ruled out. For typical values of the higgsino mass splittings, the bound extends up to the LEP kinematic limit. The higgsino masses are typically of order $|\mu|$, where the $\mu$ parameter, familiar from the MSSM, is given in our notation by

$$
\mu \equiv \frac{\lambda s}{\sqrt{2}}
$$

Thus the chargino mass limit constrains the product of $\lambda$ and $s$. The exact bound depends on the amount of mixing between the neutralinos, which again would require us to specify the gaugino masses within a particular model. It also depends on the particular framework of supersymmetry breaking, e.g. in models with low-scale supersymmetry breaking the bound from prompt decays of a higgsino NLSP [17 from the Tevatron can be stronger. We therefore again choose to stay on a modelindependent path, and we consider (rather conservatively) any value of $\mu \gtrsim 100 \mathrm{GeV}$ as allowed.

Searches for the superpartners of the two neutral $S U(2)$-singlet Higgs bosons (i.e. "singlinos") are quite challenging. The couplings of the singlinos to gauge bosons are suppressed by the neutralino mixing angles, and there are practically no limits coming from direct singlino production or $Z$-decays [18. The constraints on a light 


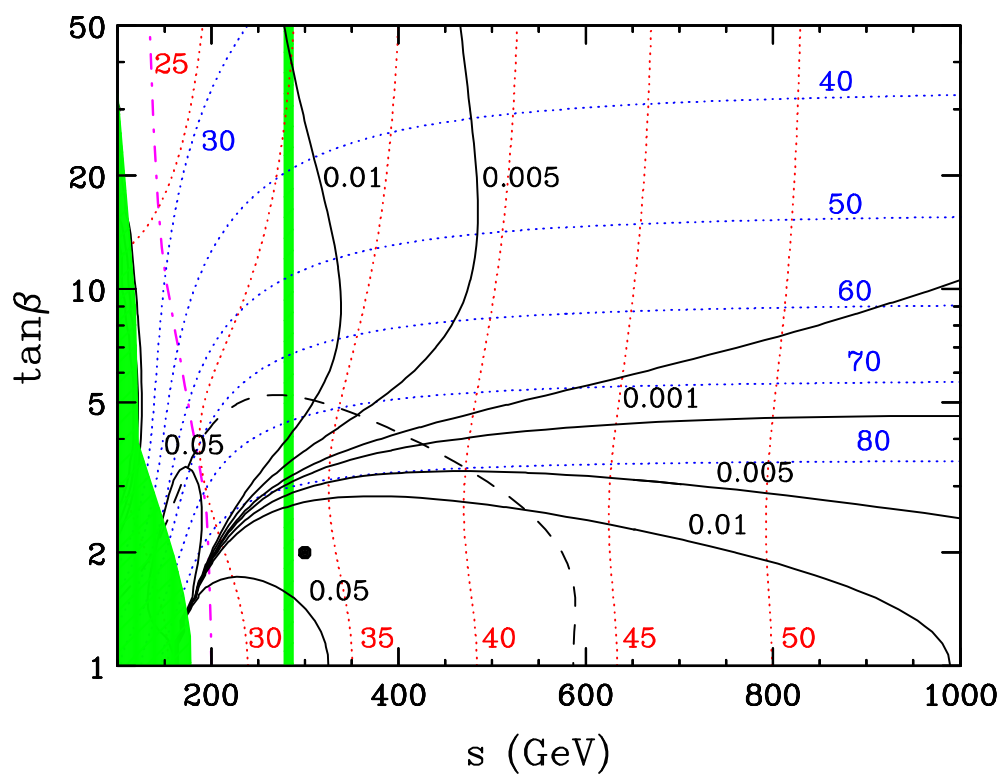

Figure 4: Higgs boson spectrum and the absolute value of the dimensionless $H_{1}^{0} A_{1}^{0} A_{1}^{0}$ coupling $c_{111}$ (see text), as a function of $s$ (in $\mathrm{GeV}$ ) and $\tan \beta$, for $\lambda=\kappa=0.5$ and $m_{\lambda}=m_{\kappa}=1 \mathrm{GeV}$. The horizontal (blue) dotted lines are contours of $M_{H_{1}^{0}}$, while the vertical grid of dotted red lines consists of $M_{A_{1}^{0}}$ iso-mass contours. The thick (green) vertical line loosely marks the assumed higgsino mass bound of $\mu=100 \mathrm{GeV}$, while the (green) shaded area contains a tachyon or is excluded from the charged Higgs search at LEP. The dot symbol denotes the values of $s$ and $\tan \beta$ used in Fig. 5. The dashed line delineates the region where $M_{H_{1}^{0}}>2 M_{A_{1}^{0}}$ at tree-level. To the right of the vertically running dotdashed (magenta) line the SM-like CP-even Higgs boson is identified with the lightest CP-even mass eigenstate: $h^{0}=H_{1}^{0}$, while to the left of the line we have $h^{0}=H_{2}^{0}$ instead. In the latter region, the corresponding coupling $c_{211}$ is larger, on the order of $10 \%$ (see the small $s$ region of Fig. 2).

CP-odd scalar with small couplings to SM fermions are also very loose [19, 20]. For example, no significant constraints on the axion mass have been set from $Z \rightarrow A^{0} \gamma$ at LEP [21], from the Yukawa process $e^{+} e^{-} \rightarrow f \bar{f} A_{1}^{0}$ [22], from direct $A^{0}$ production through gluon fusion at the Tevatron [23], from fits to the electroweak data [24], or from meson decays [25]. The LEP searches for $Z^{*} \rightarrow h^{0} A^{0} \rightarrow A^{0} A^{0} A^{0}$ within the MSSM [26] can be reinterpreted as axion searches, but the limits are diluted because the axion coupling to the $Z h^{0}$ in our case is smaller by a factor of $\cos \theta_{A}$ [see Eq. (3.5)]. The relevant lower bounds on $M_{A}$ currently come from beam dump experiments [27], in the $\mathrm{MeV}$ range, and from star cooling rates, $M_{A} \gtrsim 0.2 \mathrm{MeV}$ [28].

Having summarized the relevant collider constraints, we now present our exact numerical results for $c$. In Fig. 团 we show contours of the masses of the SM-like Higgs boson, $M_{h^{0}}$, [horizontal dotted (blue) lines] and the lightest CP-odd Higgs boson, 
$M_{A_{1}^{0}}$, [vertical dotted (red) lines] as a function of $s$ and $\tan \beta$, for fixed $\lambda=\kappa=0.5$ and $m_{\lambda}=m_{\kappa}=1 \mathrm{GeV}$. The thick (green) vertical line denotes the assumed higgsino mass bound of $\mu=100 \mathrm{GeV}$ and the region to its left is disfavored. Inside the (green) shaded area there is either a tachyon in the spectrum, or the charged Higgs mass is below the experimental bound. While it is in principle possible that the radiative corrections to the Higgs boson masses, neglected here, may shrink, or even eliminate this excluded region, we do not find this latter part of parameter space particularly attractive, since it is associated with very light higgsinos.

Throughout most of the parameter space shown in Fig. 目, the SM-like Higgs boson is the lightest CP-even mass eigenstate: $h^{0}=H_{1}^{0}$. However, at small values of $s, H^{0}$ and $H^{\prime 0}$ become light as well (see Fig. 1). The dotdashed (magenta) line going vertically marks the point at which the SM-like Higgs boson changes its identity from $h^{0}=H_{1}^{0}$ (to the right of the line) to $h^{0}=H_{2}^{0}$ (to the left).

In Fig. 4 we only show the masses of the $H_{1}^{0}$ and $A_{1}^{0}$ Higgs bosons, since we are interested in the phenomenology of the $h^{0} \rightarrow A_{1}^{0} A_{1}^{0}$ decay. The first important result seen in Fig. $⿴$ is the location of the region of parameter space, where this decay is open. The dashed line in Fig. $⿴$ delineates the relevant part of parameter space where $M_{H_{1}^{0}}>2 M_{A_{1}^{0}}$ at tree-level. The location of this region is easily understood. Recall that $M_{A_{1}^{0}}$ scales with $\sqrt{s}$, as evident from the Figure, as well as Eq. (3.1). Then notice that unlike the case of the MSSM, here the lightest CP-even Higgs boson mass decreases with $\tan \beta$, so that $\tan \beta$ values as low as $1-3$ are possible. Combining these two observations, we easily see that the $h^{0} \rightarrow A_{1}^{0} A_{1}^{0}$ decay is most likely to be open at small $\tan \beta$ and small $s$.

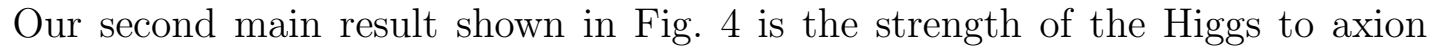
coupling. We show contours of the absolute value of the dimensionless coupling $\left|c_{111}\right|$, which we define in analogy to Eq. (3.7):

$$
\mathcal{L}_{H_{i}^{0} A_{j}^{0} A_{k}^{0}}=\frac{v}{2} \sum_{i, j, k} c_{i j k} H_{i}^{0} A_{j}^{0} A_{k}^{0} .
$$

As we already explained above, to the right of the vertical dotdashed magenta line, $c_{111}$ is identical to the coefficient $c$ defined in Eq. (3.7). We see that in the region of parameter space, free of any experimental constraints, $c$ can be as large as 0.05 . We also see the possibility of exact cancellation and vanishing $c$. Indeed, in the limit of large $s$, our leading order approximation Eq. (4.11) vanishes for $\tan \beta=2+\sqrt{3} \sim 3.7$, in reasonable agreement with Fig. 6 . In the small $s$ region, where $h^{0}=H_{2}^{0}$, the corresponding $c_{211}$ can be even larger, on the order of $10 \%$ or more.

In summary, our main conclusion from Fig. 1 is that for fixed $\lambda$ and $\kappa$, values of $c$ are typically maximized at small $s$ and small $\tan \beta$ - exactly in the spot where the $h^{0} \rightarrow A_{1}^{0} A_{1}^{0}$ channel is most likely to be open. This could also have been anticipated 


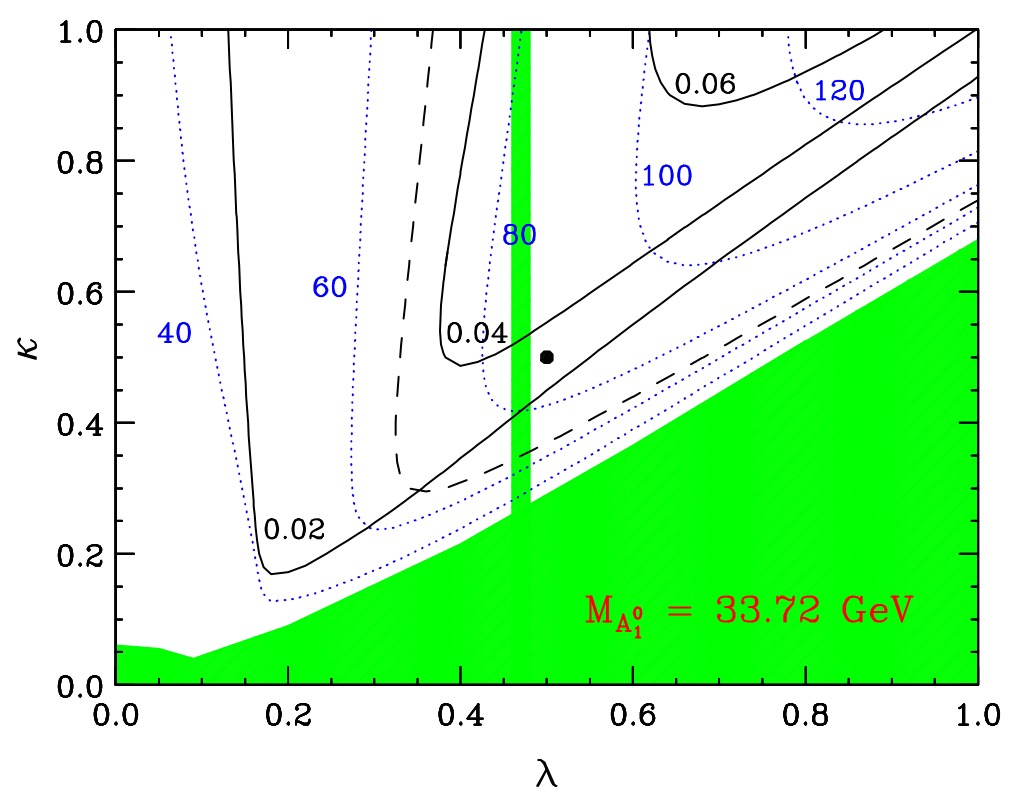

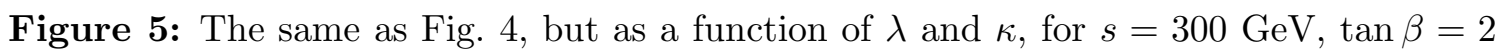
and $m_{\lambda}=m_{\kappa}=1 \mathrm{GeV}$. The dot symbol shows the values of $\lambda$ and $\kappa$ used in Fig. 4 .

from the approximate scaling $c \sim M_{h^{0}}^{2}$, which we found in the two limiting cases considered earlier.

We now turn our attention to the dependence on the other two main parameters: $\lambda$ and $\kappa$. To this end, we fix $s=300 \mathrm{GeV}$ and $\tan \beta=2$, and show the Higgs spectrum and $\left|c_{111}\right|$ in Fig. 5 as a function of $\lambda$ and $\kappa$, again with fixed $m_{\lambda}=m_{\kappa}=1 \mathrm{GeV}$. The (green) shaded area is again excluded because of tachyonic or light charged Higgs states, and the (green) vertical line denotes the assumed higgsino bound $\mu=$ $100 \mathrm{GeV}$, with the region to the left of it being disfavored. This time the SM-like Higgs boson is unambiguously identified as $h^{0}=H_{1}^{0}$. On the other hand, the axion mass is completely fixed in terms of $m_{\lambda}, m_{\kappa}, s$ and $\tan \beta$ [recall the discussion after Eq. (3.3)]. For the values of the parameters considered here, $M_{A_{1}^{0}}=33.72 \mathrm{GeV}$ and its fractional variation throughout the whole Figure is less than 1 part in $10^{5}$. The dashed line, depicting the region $M_{H_{1}^{0}}>2 M_{A_{1}^{0}}$, is therefore coincident with the contour of $M_{H_{1}^{0}}=67.44 \mathrm{GeV}$.

We see from Fig. 5 that large ratios of $\lambda / \kappa$ are disfavored. This can be easily understood from Eq. (4.3) - the second term gives a large negative contribution to the lightest CP-even Higgs boson mass, which results in $M_{h^{0}}^{2}<0$. Nevertheless, there is a significant allowed region with naturally large couplings $\lambda$ and $\kappa$. Now the lightest CP-even Higgs boson mass can be larger than the LEP limit already at tree-level. The coefficient $c$ is again maximized in the region with the largest $M_{h^{0}}$, and again values of $c \sim 0.05$ are possible. 


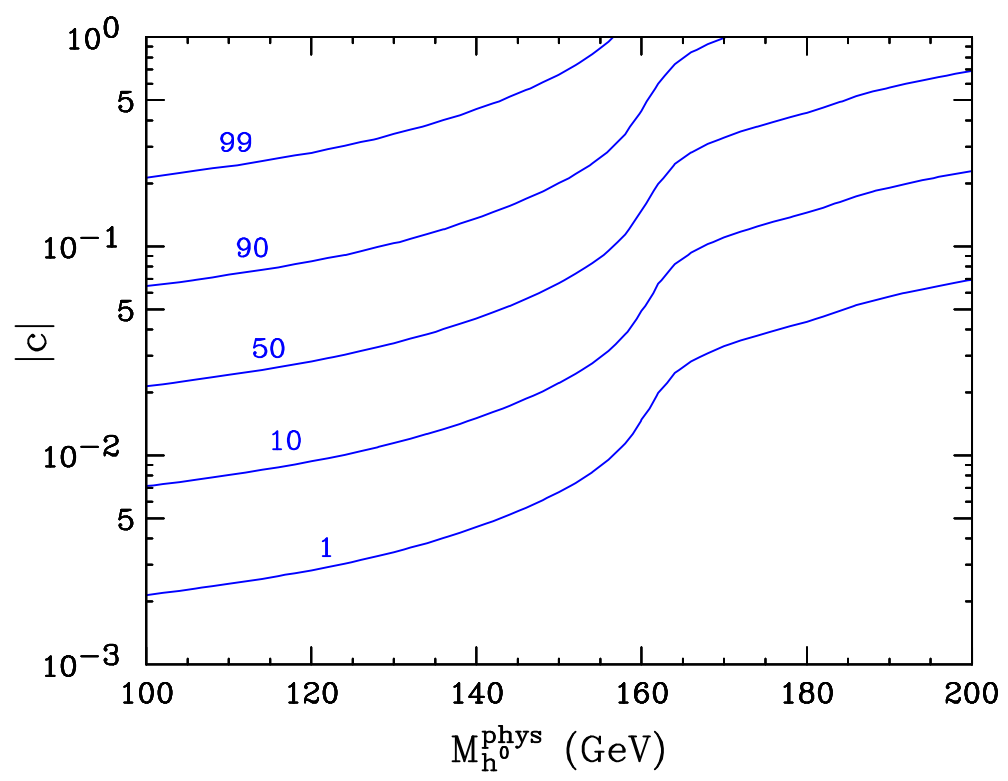

Figure 6: The branching ratio $B\left(h^{0} \rightarrow A_{1}^{0} A_{1}^{0}\right)$ in percent, as a function of the physical Higgs mass $M_{h^{0}}^{\text {phys }}$ and the value of the coefficient $c$, assuming $M_{A_{1}^{0}} \ll M_{h^{0}}^{\text {phys }}$.

Given that the small axion mass allows for the $h^{0} \rightarrow A_{1}^{0} A_{1}^{0}$ decays in principle, and that the coefficient $c$ can be sizable (see Figs. (1 and 5), it is interesting to quantify how the branching fractions of the SM-like Higgs boson, $h^{0}$, are affected. To this end, one must use the physical mass $M_{h^{0}}^{\text {phys }}$, as the partial widths for $h^{0} \rightarrow A_{1}^{0} A_{1}^{0}$ and $h^{0} \rightarrow b \bar{b}$ have the opposite dependence on $M_{h^{0}}^{\text {phys }}$. So, by using the (smaller) tree-level mass, one would be overestimating $\Gamma\left(h^{0} \rightarrow A_{1}^{0} A_{1}^{0}\right)$ and underestimating $\Gamma\left(h^{0} \rightarrow b \bar{b}\right)$. This is why in Fig. 6 we show the branching ratio for $h^{0} \rightarrow A_{1}^{0} A_{1}^{0}$ decays

$$
B\left(h^{0} \rightarrow A_{1}^{0} A_{1}^{0}\right)=\frac{\Gamma\left(h^{0} \rightarrow A_{1}^{0} A_{1}^{0}\right)}{\Gamma_{\mathrm{SM}}+\Gamma\left(h^{0} \rightarrow A_{1}^{0} A_{1}^{0}\right)},
$$

as a function of the physical Higgs mass $M_{h^{0}}^{\text {phys }}$ and the value of the coefficient $c$, assuming $M_{A_{1}^{0}} \ll M_{h^{0}}^{\text {phys }}$ and $M_{A_{2}^{0}} \gg M_{h^{0}}^{\text {phys }}$. Here we have taken $\Gamma_{\text {SM }}$ as the width of the SM Higgs boson. Fig. 6 should be interpreted as follows. After fixing the fundamental input parameters $\lambda, \kappa, m_{\lambda}, m_{\kappa}, s$ and $\tan \beta$, one should compute the physical Higgs boson mass $M_{h^{0}}^{\text {phys }}$ in terms of the parameters of the squark sector derived within a given model. Then the coefficient $c$ can be read off from Figs. 4 and 5, and the corresponding branching fraction $B\left(h^{0} \rightarrow A_{1}^{0} A_{1}^{0}\right)$ for the resulting values of $c$ and $M_{h^{0}}^{\text {phys }}$ is given in Fig. 6.

From Fig. 6 we can see the potential importance of the $h^{0} \rightarrow A_{1}^{0} A_{1}^{0}$ decay mode. In the theoretically preferred mass range $M_{h^{0}}^{\text {phs }} \lesssim 160-180 \mathrm{GeV}$ of the NMSSM, this decay mode completely dominates for $c \gtrsim 0.1$. We have seen that such values of $c$ are in principle possible, although in limited regions of parameter space. For 
the more typical values of $c \sim 0.02-0.05$, the Higgs boson branching fraction into axions is comparable, if not larger, than $B(h \rightarrow b \bar{b})$, and may still significantly affect the Higgs boson collider searches utilizing the $b \bar{b}$ mode.

\subsection{Theoretical prejudice}

The theoretical constraints discussed in this subsection are less robust, since one must define a framework. For example, when requiring the absence of Landau poles for the Higgs couplings $\lambda$ and $\kappa$, one must specify the high-energy scale $\Lambda$, at which the effective theory description of the NMSSM breaks down. Naturally, the constraints would be stronger, if $\Lambda$ is identified with the GUT or Planck scale, rather than some intermediate scale, related to the (mediation of) supersymmetry breaking.

The Renormalization Group equations (RGE's) for the Yukawa couplings of the NMSSM have been extensively studied in relation to the question of the absolute upper limit on the lightest CP-even Higgs mass [29, 30]. If $\Lambda$ is identified with the GUT scale, the perturbativity requirement implies $\lambda \lesssim 0.87$ and $\kappa \lesssim 0.63$ [12, 31, 29, 20]. If, however, $\Lambda$ is identified with an intermediate scale, as in gaugemediated models [10, 32], then the bounds can be somewhat relaxed, e.g. $\kappa \lesssim 1.36$ for $\Lambda \sim 50-100 \mathrm{TeV}$ [10]. We see that these constraints still leave a lot of the available parameter space in Figs. 1 and 5, where the new decay $h^{0} \rightarrow A_{1}^{0} A_{1}^{0}$ is possible, and its branching ratio is sizable.

One may also wonder if the low values of $m_{\lambda}$ and $m_{\kappa}$ needed in order to suppress the axion mass $M_{A_{1}}$ (see Eq. (3.1)) can appear naturally, without any significant finetuning. So far we have adopted a low-energy point of view and never specified the particular model framework for supersymmetry breaking and its communication to the NMSSM sector. The size of $m_{\lambda}$ and $m_{\kappa}$ will depend on two factors: the boundary conditions at the high-energy scale $\Lambda$, where the soft supersymmetry breaking parameters are generated, and second, the amount of (logarithmic) RGE running from that scale down to the electroweak scale $v$. Notice that due to the singlet nature of $\hat{S}$, the one-loop beta function for $m_{\kappa}$ only depends on $m_{\lambda}$ and $m_{\kappa}$. In the $U(1)_{R}$ limit both of these two parameters start out small at the high-energy scale $\Lambda$, and the generated value for $m_{\kappa}$ at the weak scale is typically also rather small. On the other hand, the one-loop beta function for $m_{\kappa}$ depends on the gaugino masses $M_{1}$ and $M_{2}$, as well as the rest of the $A$-term parameters, which can be relatively large already at the scale $\Lambda$. Thus the induced value for $m_{\lambda}$ at the weak scale can be much larger than $m_{\kappa}$. However, a closer inspection of Eq. (3.1) reveals that the axion mass is usually more sensitive to $m_{\kappa}$ rather than $m_{\lambda}$. For example, in the large $\tan \beta$ limit we have $\cos \theta_{A} \sim 1 / \tan \beta$, hence

$$
M_{A_{1}^{0}}^{2} \sim \mathcal{O}\left(m_{\kappa} s\right)+\mathcal{O}\left(\frac{m_{\lambda} s}{\tan \beta}\right) \sim \mathcal{O}\left(m_{\kappa} s\right) .
$$


Without a particular model, it is difficult to be more specific at this point. We shall therefore leave this question open for future studies. Let us only point out that there exist at least two very well motivated frameworks, in which the boundary conditions for $m_{\lambda}$ and $m_{\kappa}$ are zero at the scale $\Lambda$ - gauge mediation [33, 34] and gaugino mediation [35].

\section{Conclusions}

We have discussed a limit of the NMSSM where a light CP-odd scalar (axion) is present in the Higgs spectrum. The light axion appears as a result of an approximate global $U(1)_{R}$ symmetry of the scalar potential, which is spontaneously broken. We found that the mass of the axion is proportional to the soft breaking trilinear couplings $m_{\lambda}$ and $m_{\kappa}$, and if those are in the GeV range, the axion can easily be lighter than half the SM-like Higgs boson mass, $M_{h}^{0}$. In those cases, we computed the $h^{0} A_{1}^{0} A_{1}^{0}$ coupling and found that it can have a direct impact on phenomenology, as it can substantially modify the SM-like Higgs boson collider signatures.

In conclusion, we feel that our results fill a major gap in the extensive literature on the Higgs sector of the NMSSM. Previous NMSSM studies have mostly concentrated on setting absolute upper limits on the SM-like Higgs boson mass [36] (which is of primary interest for the production of $h^{0}$ in collider experiments) or the related singlino phenomenology 18, 37]. However, the case of a light CP-odd axion considered here has largely been overlooked. In light of the interesting phenomenological implications of the scenario presented here, and the symmetry reasons behind its motivation, it is worth pursuing the case of a light CP-odd scalar in future phenomenological studies.

\section{Acknowledgments}

We thank M. Carena, H.-C. Cheng, S. Mrenna, A. Pilaftsis, M. Schmaltz and C. Wagner for comments and questions. Fermilab is operated under DOE contract DEAC02-76CH03000.

\section{Appendix: Higgs Coupling to Axions in Polar Field Coordi- nates}

In this Appendix we derive the Higgs boson coupling to axion pairs using the polar field coordinates. Although the physical results are independent of the parametrization of the degrees of freedom, and the orthogonal field coordinates used in section 
2 [see Eq. (2.5)] are convenient enough, the polar field coordinates are particularly appropriate for describing the axion in the limit where we neglect its mass.

Consider the following parametrization:

$$
\begin{aligned}
& H_{d}=\left(\begin{array}{c}
\frac{1}{\sqrt{2}}\left[\left(v+h_{v}^{0}\right) \cos \beta-H_{v}^{0} \sin \beta\right] e^{i\left(A_{v}^{0} \tan \beta-G^{0}\right) / v} \\
-G^{-} \cos \beta+H^{-} \sin \beta
\end{array}\right), \\
& H_{u}=\left(\begin{array}{c}
G^{+} \sin \beta+H^{+} \cos \beta \\
\frac{1}{\sqrt{2}}\left[\left(v+h_{v}^{0}\right) \sin \beta+H_{v}^{0} \cos \beta\right] e^{i\left(A_{v}^{0} \cot \beta+G^{0}\right) / v}
\end{array}\right), \\
& S=\frac{1}{\sqrt{2}}\left(s+h_{s}^{0}\right) e^{i A_{s}^{0} / s}
\end{aligned}
$$

With this parametrization, it is straightforward to derive the masses and mixing angle of the CP-odd states obtained in section 3.2.

More importantly, the scalar potential Eq. (2.5) does not involve the axion field in the $m_{\lambda, \kappa} \rightarrow 0$ limit. If we neglect the axion mass, then only the kinetic terms give rise to axion couplings. This is the usual statement that a Nambu-Goldstone boson has derivative couplings in the polar field coordinate parametrization. The kinetic terms for $H_{u}, H_{d}$ and $S$ include canonically normalized kinetic terms for the physical states as well as derivative couplings of CP-odd scalar pairs with CP-even scalars. The SM-like Higgs boson coupling to axion pairs can be easily derived:

$$
\mathcal{L}_{h^{0} A_{1}^{0} A_{1}^{0}}^{\prime}=\frac{c^{\prime} v}{2 M_{h}^{2}} h^{0} \partial_{\mu} A_{1}^{0} \partial^{\mu} A_{1}^{0},
$$

where the dimensionless parameter $c^{\prime}$ is given by

$$
c^{\prime}=2 \frac{M_{h}^{2}}{v^{2}}\left[\left(U_{11}+2 U_{12} \cot 2 \beta\right) \cos ^{2} \theta_{A}+\frac{v}{s} U_{13} \sin ^{2} \theta_{A}\right]
$$

In the $s \gg v$ limit, the elements of the matrix $U$ that rotates the CP-even scalars to the mass eigenbasis have been computed in section 4. Using Eq. (4.1) we find a very simple result:

$$
c^{\prime}=-2 c\left[1+\mathcal{O}\left(v / s, m_{\lambda, \kappa} / s\right)\right],
$$

where $c$ is given by Eq. (4.11). The last step of this computation is to check that the decay width for $h^{0} \rightarrow A_{1}^{0} A_{1}^{0}$ is the same as the one obtained using orthogonal coordinates [see Eq. (4.10)]. This straigthforward exercise provides the explanation for the $M_{h}^{2} / s^{2}$ dependence of $c$ in Eq. (4.11). 


\section{References}

[1] B. A. Dobrescu, G. Landsberg and K. T. Matchev, "Higgs boson decays to CP-odd scalars at the Tevatron and beyond," hep-ph/0005308.

[2] H. P. Nilles, M. Srednicki and D. Wyler, "Weak Interaction Breakdown Induced By Supergravity," Phys. Lett. B120, 346 (1983); J. M. Frere, D. R. Jones and S. Raby, "Fermion Masses And Induction Of The Weak Scale By Supergravity," Nucl. Phys. B222, 11 (1983); J. P. Derendinger and C. A. Savoy, "Quantum Effects And $S U(2) \times U(1)$ Breaking In Supergravity Gauge Theories," Nucl. Phys. B237, 307 (1984).

[3] B. A. Dobrescu, "Minimal composite Higgs model with light bosons," hep$\mathrm{ph} / 9908391$.

[4] R. E. Shrock and M. Suzuki, "Invisible Decays Of Higgs Bosons," Phys. Lett. B110, 250 (1982); L. Li, Y. Liu and L. Wolfenstein, "Hidden Higgs Particles," Phys. Lett. B159, 45 (1985); A. S. Joshipura and S. D. Rindani, "Majoron models and the Higgs search," Phys. Rev. Lett. 69, 3269 (1992).

[5] H. Cheng, B. A. Dobrescu and K. T. Matchev, "A chiral supersymmetric standard model," Phys. Lett. B439, 301 (1998) hep-ph/9807246; H. Cheng, B. A. Dobrescu and K. T. Matchev, "Generic and chiral extensions of the supersymmetric standard model," Nucl. Phys. B543, 47 (1999) hep-ph/9811316; J. Erler, "Chiral models of weak scale supersymmetry," hep-ph/0006051.

[6] G. K. Yeghian, "Upper bound on the lightest Higgs mass in supersymmetric theories," Acta Physica Slovaka 49, 823 (1999), hep-ph/9904488.

[7] F. Franke and H. Fraas, "Neutralinos and Higgs Bosons in the Next-To-Minimal Supersymmetric Standard Model," Int. J. Mod. Phys. A12, 479 (1997) hepph/9512366.

[8] A. E. Nelson and N. Seiberg, "R symmetry breaking versus supersymmetry breaking," Nucl. Phys. B416, 46 (1994) hep-ph/9309299.

[9] J. Bagger, E. Poppitz and L. Randall, "The R axion from dynamical supersymmetry breaking," Nucl. Phys. B426, 3 (1994) hep-ph/9405345.

[10] T. Han, D. Marfatia and R. Zhang, "A gauge-mediated supersymmetry breaking model with an extra singlet Higgs field," Phys. Rev. D61, 013007 (2000) hepph/9906508.

[11] M. Drees, "Supersymmetric Models With Extended Higgs Sector," Int. J. Mod. Phys. A4, 3635 (1989). 
[12] J. Ellis, J. F. Gunion, H. E. Haber, L. Roszkowski and F. Zwirner, "Higgs Bosons In A Nonminimal Supersymmetric Model," Phys. Rev. D39, 844 (1989).

[13] Peter Igo-Kemenes, "Results from $e^{+} e^{-}$Colliders ", talk given at the "ICHEP2000" conference, July 27 - August 2, 2000, Osaka, Japan.

[14] H. E. Haber and R. Hempfling, "Can the mass of the lightest Higgs boson of the minimal supersymmetric model be larger than $m(Z)$ ?," Phys. Rev. Lett. 66, 1815 (1991).

[15] U. Ellwanger, "Radiative corrections to the neutral Higgs spectrum in supersymmetry with a gauge singlet," Phys. Lett. B303, 271 (1993) hep-ph/9302224; T. Elliott, S. F. King and P. L. White, "Squark contributions to Higgs boson masses in the next-to-minimal supersymmetric standard model," Phys. Lett. B314, 56 (1993) hepph/9305282]; T. Elliott, S. F. King and P. L. White, "Radiative corrections to Higgs boson masses in the next-to-minimal supersymmetric Standard Model," Phys. Rev. D49, 2435 (1994) hep-ph/9308309; U. Ellwanger and C. Hugonie, "Masses and couplings of the lightest Higgs bosons in the (M+1)SSM," hep-ph/9909260.

[16] G. Grenier, " Almost degenerate chargino-neutralino at LEP", talk given at the "SUSY2K" conference, CERN, Geneva, Switzerland, 26 June - 1 July 2000.

[17] H. Baer, P. G. Mercadante, X. Tata and Y. Wang, "The reach of Tevatron upgrades in gauge-mediated supersymmetry breaking models," Phys. Rev. D60, 055001 (1999) hep-ph/9903333; K. T. Matchev and S. Thomas, "Higgs and Z-boson signatures of supersymmetry," hep-ph/9908482.

[18] F. Franke and H. Fraas, "Production and decay of neutralinos in the next-to-minimal supersymmetric standard model," Z. Phys. C72, 309 (1996) hep-ph/9511275; U. Ellwanger and C. Hugonie, "Topologies of the $(\mathrm{M}+1) \mathrm{SSM}$ with a singlino LSP at LEP2," Eur. Phys. J. C13, 681 (2000) hep-ph/9812427]; S. Hesselbach, F. Franke and H. Fraas, "Neutralino production with polarized beams in extended supersymmetric models," hep-ph/0003272.

[19] F. Franke and H. Fraas, "Mass bounds for the neutral Higgs bosons in the next-to-minimal supersymmetric standard model," Phys. Lett. B353, 234 (1995) hep-ph/9504279]; U. Ellwanger, M. Rausch de Traubenberg, C. Strasbourg and C. A. Savoy, "Higgs phenomenology of the supersymmetric model with a gauge singlet," Z. Phys. C67, 665 (1995) hep-ph/9502206.

[20] S. F. King and P. L. White, "Nonminimal supersymmetric Higgs bosons at LEP-2," Phys. Rev. D53, 4049 (1996) hep-ph/9508346.

[21] S. Raychaudhuri and A. Raychaudhuri, "Singlet Higgs boson signals at electron positron colliders," Phys. Rev. D44, 2663 (1991); G. Rupak and E. H. Simmons, 
"Limits on pseudoscalar bosons from rare Z decays at LEP," Phys. Lett. B362, 155 (1995) hep-ph/9507438]; M. Krawczyk, J. Zochowski and P. Mattig, "Process $Z \rightarrow h(A)+\gamma$ in the 2 HDM and the experimental constraints from LEP," Eur. Phys. J. C8, 495 (1999) hep-ph/9811256.

[22] A. Djouadi, P. M. Zerwas and J. Zunft, "Search for light pseudoscalar Higgs bosons in Z decays," Phys. Lett. B259, 175 (1991); J. Kalinowski and M. Krawczyk, "Fermion mass effects on $\Gamma(Z \longrightarrow b \bar{b}+$ a light Higgs $)$ in a two Higgs doublet model," Phys. Lett. B361, 66 (1995) hep-ph/9506291.

[23] A. Datta, A. Raychaudhuri, S. Raychaudhuri and S. Chakrabarti, "Singlet-Higgsboson signals at hadron colliders," Z. Phys. C72, 449 (1996) hep-ph/9510432.

[24] P. H. Chankowski, M. Krawczyk and J. Zochowski, "Implications of the precision data for very light Higgs boson scenario in 2HDM(II)," Eur. Phys. J. C11, 661 (1999).

[25] J. F. Gunion, H. E. Haber, G. Kane and S. Dawson, "The Higgs Hunter's Guide", Addison-Wesley, 1996.

[26] O. Adriani et al. [L3 Collaboration], "Searches for nonminimal Higgs bosons in Z0 decays," Phys. Lett. B294, 457 (1992); K. Ackerstaff et al. [OPAL Collaboration], "A search for neutral Higgs bosons in the MSSM and models with two scalar field doublets," Eur. Phys. J. C5, 19 (1998) hep-ex/9803019; G. Abbiendi et al., [OPAL Collaboration], "Search for neutral Higgs bosons in $e^{+} e^{-}$ collisions at $s^{1 / 2} \approx 189 \mathrm{GeV}$," hep-ex/9908002; OPAL Physics Note PN-416, www.cern.ch/Opal/pubs/physnote/html/pn416.html.

[27] J. Blumlein et al., "Limits on neutral light scalar and pseudoscalar particles in a proton beam dump experiment," Z. Phys. C51, 341 (1991).

[28] D. E. Groom et al., "Review of particle physics," Eur. Phys. J. C15 (2000) 1.

[29] W. T. ter Veldhuis, "Mass of the lightest Higgs boson in the minimal supersymmetric Standard Model with an additional singlet," hep-ph/9211281.

[30] U. Ellwanger and M. Lindner, "Constraints on new physics from the Higgs and top masses," Phys. Lett. B301, 365 (1993) hep-ph/9211249; U. Ellwanger, M. Rausch de Traubenberg and C. A. Savoy, "Particle spectrum in supersymmetric models with a gauge singlet," Phys. Lett. B315, 331 (1993), hep-ph/9307322; T. Elliott, S. F. King and P. L. White, "Unification constraints in the next-to-minimal supersymmetric standard model," Phys. Lett. B351, 213 (1995) hep-ph/9406303.

[31] J. R. Espinosa and M. Quiros, "On Higgs boson masses in nonminimal supersymmetric standard models," Phys. Lett. B279, 92 (1992). 
[32] A. de Gouvea, A. Friedland and H. Murayama, "Next-to-minimal supersymmetric standard model with the gauge mediation of supersymmetry breaking," Phys. Rev. D57, 5676 (1998) hep-ph/9711264; M. Masip, R. Munoz-Tapia and A. Pomarol, "Limits on the mass of the lightest Higgs in supersymmetric models," Phys. Rev. D57, 5340 (1998) hep-ph/9801437.

[33] M. Dine and A. E. Nelson, "Dynamical supersymmetry breaking at low-energies," Phys. Rev. D48, 1277 (1993) hep-ph/9303230; M. Dine, A. E. Nelson and Y. Shirman, "Low-energy dynamical supersymmetry breaking simplified," Phys. Rev. D51, 1362 (1995) hep-ph/9408384; M. Dine, A. E. Nelson, Y. Nir and Y. Shirman, "New tools for low-energy dynamical supersymmetry breaking," Phys. Rev. D53, 2658 (1996) [hep-ph/9507378.

[34] S. Dimopoulos, S. Thomas and J. D. Wells, "Sparticle spectroscopy and electroweak symmetry breaking with gauge-mediated supersymmetry breaking," Nucl. Phys. B488, 39 (1997) hep-ph/9609434; J. A. Bagger, K. Matchev, D. M. Pierce and R. Zhang, "Weak-scale phenomenology in models with gauge-mediated supersymmetry breaking," Phys. Rev. D55, 3188 (1997) hep-ph/9609444.

[35] D. E. Kaplan, G. D. Kribs and M. Schmaltz, "Supersymmetry breaking through transparent extra dimensions," hep-ph/9911293; Z. Chacko, M. A. Luty, A. E. Nelson and E. Ponton, "Gaugino mediated supersymmetry breaking," JHEP 0001, 003 (2000) hep-ph/9911323]; M. Schmaltz and W. Skiba, "Minimal gaugino mediation," hep-ph/0001172; M. Schmaltz and W. Skiba, "The superpartner spectrum of gaugino mediation," hep-ph/0004210.

[36] U. Ellwanger and M. Rausch de Traubenberg, "Natural range of Higgs masses in supersymmetry," Z. Phys. C53, 521 (1992); J. R. Espinosa and M. Quiros, "Upper bounds on the lightest Higgs boson mass in general supersymmetric Standard Models," Phys. Lett. B302, 51 (1993) hep-ph/9212305; T. Elliott, S. F. King and P. L. White, "Supersymmetric Higgs bosons at the limit," Phys. Lett. B305, 71 (1993) hep-ph/9302202; J. Kamoshita, Y. Okada and M. Tanaka, "Neutral scalar Higgs masses and production cross-sections in an extended supersymmetric Standard Model," Phys. Lett. B328, 67 (1994) hep-ph/9402278; P. N. Pandita, "Upper bound on the mass of lightest higgs boson in the supersymmetric singlet majoron model," Mod. Phys. Lett. A10, 1533 (1995) hep-ph/9412247]; J. R. Espinosa, "The supersymmetric singlet majoron model and the general upper bound on the lightest Higgs boson mass," Phys. Lett. B353, 243 (1995) hep-ph/9503255; B. Ananthanarayan and P. N. Pandita, "The nonminimal supersymmetric standard model with $\tan \beta \approx m_{t} / m_{b}$," Phys. Lett. B353, 70 (1995) [hep-ph/9503323]; S. F. King and P. L. White, "Resolving the constrained minimal and next-to-minimal supersymmetric standard models," Phys. Rev. D52, 4183 (1995) hep-ph/9505326; G. M. Asatrian and G. K. Egiian, "Higgs boson masses in supersymmetric theories," Mod. 
Phys. Lett. A10, 2943 (1995) hep-ph/9508263]; B. Ananthanarayan and P. N. Pandita, "Particle Spectrum in the Non-Minimal Supersymmetric Standard Model with $\tan \beta \simeq m_{t} / m_{b}$," Phys. Lett. B371, 245 (1996) hep-ph/9511415; B. Ananthanarayan and P. N. Pandita, "The Non-Minimal Supersymmetric Standard Model at Large $\tan \beta$," Int. J. Mod. Phys. A12, 2321 (1997) hep-ph/9601372; S. W. Ham, S. K. Oh and B. R. Kim, "Absolute Upper Bound on the 1-loop Corrected mass of $S_{1}$ in the NMSSM," J. Phys. G G22, 1575 (1996) hep-ph/9604243; D. Comelli and J. R. Espinosa, "New limits on the mass of neutral Higgses in general models," Phys. Lett. B388, 793 (1996) hep-ph/9607400; U. Ellwanger, M. Rausch de Traubenberg and C. A. Savoy, "Phenomenology of supersymmetric models with a singlet," Nucl. Phys. B492, 21 (1997) hep-ph/9611251; S. W. Ham, S. K. Oh and B. R. Kim, "Experimental constraints on the parameter space of the next-to-minimal supersymmetric standard model at LEP-2," Phys. Lett. B414, 305 (1997) hep-ph/9612294;

U. Ellwanger and C. Hugonie, "Higgs masses and couplings in the NMSSM," hep$\mathrm{ph} / 0006222$.

[37] U. Ellwanger and C. Hugonie, "Neutralino cascades in the (M+1)SSM," Eur. Phys. J. C5, 723 (1998) hep-ph/9712300; U. Ellwanger and C. Hugonie, "Cascade decays in the NMSSM," hep-ph/9901309. 